\title{
The Discrete Voronoi Game in a Simple Polygon
}

\author{
Aritra Banik ${ }^{1}$, Sandip Das ${ }^{1}$, Anil Maheshwari ${ }^{2}$, and Michiel Smid ${ }^{2}$ \\ ${ }^{1}$ Indian Statistical Institute, Kolkata 700108, India \\ \{aritra.banik, sandip.das.69\}@gmail.com \\ ${ }^{2}$ School of Computer Science, Carleton University, Ottawa, ON K1S 5B6, Canada. \\ $\{$ anil, michiel\}@scs. carleton.ca
}

\begin{abstract}
Let $P$ be a simple polygon with $m$ vertices and let $\mathcal{U}$ be a set of $n$ points in $P$. We consider the points of $\mathcal{U}$ to be "users". We consider a game with two players $\mathcal{P}_{1}$ and $\mathcal{P}_{2}$. In this game, $\mathcal{P}_{1}$ places a point facility inside $P$, after which $\mathcal{P}_{2}$ places another point facility inside $P$. We say that a user $u \in \mathcal{U}$ is served by its nearest facility, where distances are measured by the geodesic distance in $P$. The objective of each player is to maximize the number of users they serve. We show that for any given placement of a facility by $\mathcal{P}_{1}$, an optimal placement for $\mathcal{P}_{2}$ can be computed in $O(m+n(\log n+\log m))$ time. We also provide a polynomial-time algorithm for computing an optimal placement for $\mathcal{P}_{1}$.
\end{abstract}

\section{Introduction}

In a facility location problem, we are interested in finding a placement of a set of facilities so that, for a given set of users, certain optimality criteria are met. In a typical geometric facility location problem, the facilities and users are modeled as points. Each user is served by its nearest facility, with respect to an appropriate distance measure (e.g., Euclidean distance). Consequently, each facility has its service zone, consisting of the set of users that are served by it. The aim is to place the facilities so that certain optimality criteria are satisfied.

The Voronoi game is a competitive facility location problem introduced by Ahn et al.[1]. Given a user space, two players, $\mathcal{P}_{1}$ and $\mathcal{P}_{2}$, sequentially place a set of point facilities. These facilities partition the user space into a set of regions, such that all users within a region are served by a particular facility. The objective of each player is to maximize the total service zone of all its facilities. This problem is generally intractable. Teramoto, Demaine, and Uehara [8] have shown that even if the underling user space is a graph, finding a winning strategy of $\mathcal{P}_{2}$ (even for a very restricted case) is NP hard. Similar results can also be found in a seminal paper of Hakimi [7].

The discrete version of the Voronoi game is studied by Banik, Battacharya and Das [3]. Their user space is a line containing a set $\mathcal{U}$ of $n$ point users. Each of the players $\mathcal{P}_{1}$ and $\mathcal{P}_{2}$ can place $k=O(1)$ point facilities. First, $\mathcal{P}_{1}$ chooses a set $F_{1}$ of $k$ facilities, after which $\mathcal{P}_{2}$ chooses a set $F_{2}$ of $k$ facilities, disjoint from $F_{1}$. The payoff of $\mathcal{P}_{2}$ is defined to be the cardinality of the set of points in $\mathcal{U}$ which are closer to some facility owned by $\mathcal{P}_{2}$ than to every facility owned by $\mathcal{P}_{1}$. The 
payoff of $\mathcal{P}_{1}$ is the number of users in $\mathcal{U}$ minus the payoff of $\mathcal{P}_{2}$. The objective of both players is to maximize their respective payoffs. Banik et al. show that, if the sorted order of points in $\mathcal{U}$ along the line is given, an optimal strategy of $\mathcal{P}_{2}$ for any given placement of facilities of $\mathcal{P}_{1}$ can be computed in linear time. They also provide results for determining an optimal strategy for $\mathcal{P}_{1}$.

Given a set of existing facilities, the problem of placing a set of new facilities, to maximize the number of users served by the new ones, has been actively researched. Cabello et al. [5] study the case when only one new facility by $\mathcal{P}_{2}$ is introduced. This problem is referred to as the MaxCov problem. They have shown that the optimal placement for the new facility can be found in $O\left(n^{2}\right)$ time. The 2-MaxCov problem, which considers the problem of placing two new facilities, has been studied by Bhattacharya and Nandy [4]. Recently Bandyapadhyay, Banik, Das and Sarkar [2] studied the one round discrete Voronoi game for graphs.

In this paper, we consider the Voronoi game, where the underlying user space is a simple polygon $P$ with distance measure defined to be the geodesic (i.e., shortest-path) distance in $P$. The game consists of a set $\mathcal{U}$ of $n$ point-users inside $P$, and two players $\mathcal{P}_{1}$ and $\mathcal{P}_{2}$. Initially, $\mathcal{P}_{1}$ places a set $F_{1}$ of $k$ point-facilities, after which $\mathcal{P}_{2}$ places a set $F_{2}$ of $k$ point-facilities, where $F_{1} \cap F_{2}=\emptyset$. Each user $u \in \mathcal{U}$ is served by the nearest facility according to the nearest neighbor rule (i.e., by the facility which is at the least geodesic distance from $u$ ).

Definition 1. (Service zone) For each facility $f \in F_{1} \cup F_{2}$, we define its service zone $\mathcal{U}_{F_{1} \cup F_{2}}(\{f\})$ to be the set of users in $\mathcal{U}$ that are closer to $f$ than to any other facility of $F_{1} \cup F_{2}$.

Given a set $S \subseteq F_{1} \cup F_{2}$, we define the service zone of $S$ to be the set of users which are assigned to one of the facilities in $S$, i.e., $\mathcal{U}_{F_{1} \cup F_{2}}(S)=$ $\cup_{f \in S} \mathcal{U}_{F_{1} \cup F_{2}}(\{f\})$.

With this definition, the problem considered in this paper can be formally described as follows.

Definition 2. Discrete Voronoi Game for a Simple Polygon P: Given a set $\mathcal{U}$ of $n$ point-users and two players $\mathcal{P}_{1}$ and $\mathcal{P}_{2}$, having $k$ facilities each, $\mathcal{P}_{1}$ chooses a set $F_{1}$ of $k$ point-facilities in $P$, after which $\mathcal{P}_{2}$ chooses a set $F_{2}$ of $k$ point-facilities in $P$, where $F_{1} \cap F_{2}=\emptyset$.

(a) Given any choice of $F_{1}$ by $\mathcal{P}_{1}$, the objective of $\mathcal{P}_{2}$ is to choose a set $S=F_{2}$ that maximizes $\mathcal{U}_{F_{1} \cup S}(S) \mid$ over all sets $S$, with $|S|=k$ and $F_{1} \cap S=\emptyset$.

(b) The objective of $\mathcal{P}_{1}$ is to place a set $F_{1}$ of $k$ facilities such that the maximum possible payoff of $\mathcal{P}_{2}$ is minimized. In other words, the objective of $\mathcal{P}_{1}$ is to choose a set $F=F_{1}$ of size $k$ that minimizes $\max _{S}\left|\mathcal{U}_{F \cup S}(S)\right|$, where the maximum is taken over all sets $S$, with $|S|=k$ and $F \cap S=\emptyset$.

In this paper, we consider the case when $k=1$. Thus, $\mathcal{P}_{1}$ will place a single facility inside $P$, after which $\mathcal{P}_{2}$ places another facility inside $P$. In the next section, we characterize an optimal placement for $\mathcal{P}_{2}$ and show that, given any 
placement of a facility by $\mathcal{P}_{1}$, an optimal strategy for $\mathcal{P}_{2}$ can be computed in $O(m+n(\log n+\log m))$ time, where $m$ is the number of vertices of $P$. In Section 3 , we will provide an algorithm that computes an optimal strategy for $\mathcal{P}_{1}$.

\section{Computing an Optimal Placement for $\mathcal{P}_{2}$}

Let $f$ (= first) and $s$ (= second) be the facilities in $P$ that are placed by players $\mathcal{P}_{1}$ and $\mathcal{P}_{2}$, respectively.

Given any placement of the facility $f$ by $\mathcal{P}_{1}$, we will provide an algorithm that computes a point $s$ that maximizes $\left|\mathcal{U}_{\{f, s\}}(\{s\})\right|$ over all points $s \in P$, where $s \neq f$.

Consider the set $\mathcal{U}=\left\{u_{1}, u_{2}, \ldots, u_{n}\right\}$ of users. For each user $u_{i}$, let $d_{i}$ denote the geodesic distance between $u_{i}$ and $f$, and let $\Gamma_{i}$ denote the set of points in the polygon $P$ whose geodesic distance to $u_{i}$ is at most $d_{i}$ (see Figure 1 ).

Observation $1 A$ user $u_{i} \in \mathcal{U}$ is served by a facility s placed by $\mathcal{P}_{2}$ if and only if $s$ belongs to $\Gamma_{i}$.

Hence, if we consider the arrangement inside $P$ defined by the set of regions $\Gamma=\left\{\Gamma_{1}, \Gamma_{2}, \ldots \Gamma_{n}\right\}$, then an optimal placement for $\mathcal{P}_{2}$ belongs to a cell in this arrangement having maximum depth.

For any two points $p_{1}$ and $p_{2}$ in $P$, denote the geodesic path between $p_{1}$ and $p_{2}$ by $\lambda\left(p_{1}, p_{2}\right)$. The length of a geodesic path $\lambda$ is denoted by $|\lambda|$. The anchor of $f$ with respect to $u_{i}$ is defined to be the last vertex on the path $\lambda\left(u_{i}, f\right)$ from $u_{i}$ to $f$; we denote this anchor by $a_{i}(f)$. If $f$ is visible from $u_{i}$ then we define $a_{i}(f)=u_{i}$. Let $C_{i}$ denote the circle centered at $a_{i}(f)$ and passing through $f$. For any two points $p_{1}, p_{2} \in P$, denote the line segment joining them by $\left[p_{1}, p_{2}\right]$.

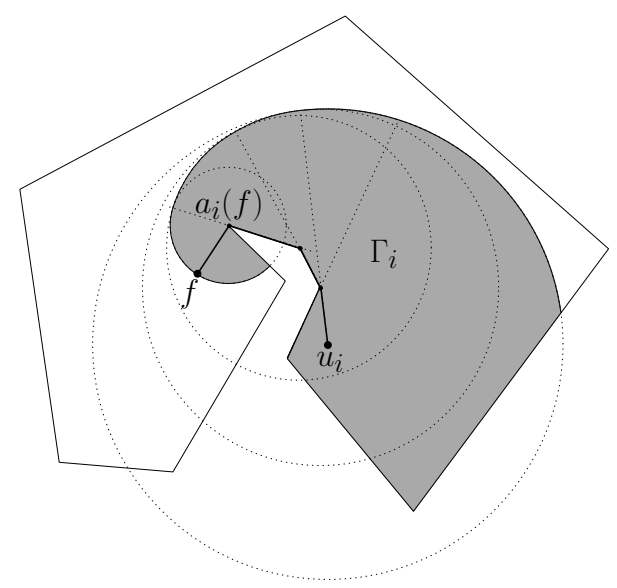

Fig. 1. Span of the user $u_{i}$ 
For any anchor vertex $a_{i}(f)$, let $l_{i}$ denote the line tangent to the circle $C_{i}$ and passing through the point $f$ (see Figure 2(a)). Consider the line segment $[c, d] \subset l_{i}$ of maximum length that is completely contained in $P$ and that contains $f$. Observe that $[c, d]$ divides the polygon into two parts. Denote the part which contains $u_{i}$ by $P_{i}$.

Lemma 1. For any user $u_{i}, \Gamma_{i} \subseteq P_{i}$.

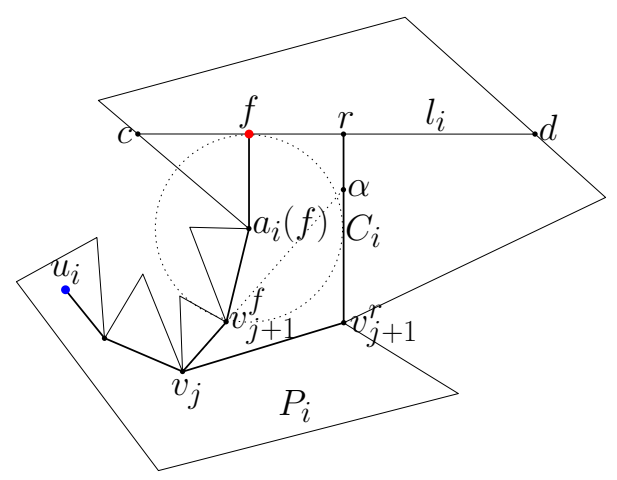

(a)

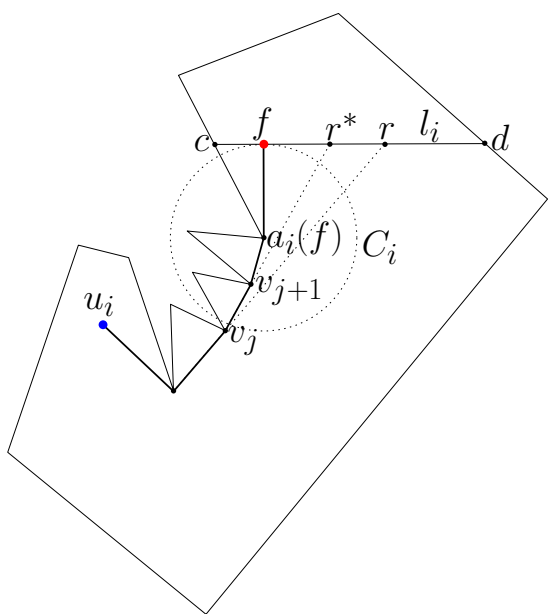

(b)

Fig. 2. Illustration of the proof of Lemma 1

Proof. If $a_{i}(f)=u_{i}$, then there is nothing to prove. In the rest of the proof, we assume that $a_{i}(f) \neq u_{i}$. It is sufficient to prove that for any point $q \in[c, d]$, $\left|\lambda\left(u_{i}, q\right)\right|>\left|\lambda\left(u_{i}, f\right)\right|$. Observe that $\left[a_{i}(f), f\right]$ divides $P_{i}$ in two parts. Without loss of generality, assume that $d$ and $u_{i}$ belong to the same sub-polygon and that $c$ belongs to the other sub-polygon. For all points $q \in[c, f]$, the shortest path from $u_{i}$ to $q$ is through $a_{i}(f)$. Hence, for all points $q \in[c, f],\left|\lambda\left(u_{i}, q\right)\right|>\left|\lambda\left(u_{i}, f\right)\right|$. Assume there exists a point $r$ in $[f, d]$, such that $\left|\lambda\left(u_{i}, r\right)\right|<\left|\lambda\left(u_{i}, f\right)\right|$. Let $r \in[f, d]$ be such a point that is closest to $f$.

Claim: The set of vertices in $\lambda\left(u_{i}, r\right)$ is a subset of the set of vertices in $\lambda\left(u_{i}, f\right)$. Proof of Claim. Let the vertices on the path $\lambda\left(u_{i}, f\right)$ be

$$
\lambda\left(u_{i}, f\right)=\left(v_{1}, v_{2}, \ldots, v_{j}, v_{j+1}^{f}, \ldots, v_{\tau}^{f}, f\right)
$$

and let the vertices on the path $\lambda\left(u_{i}, r\right)$ be

$$
\lambda\left(u_{i}, r\right)=\left(v_{1}, v_{2}, \ldots, v_{j}, v_{j+1}^{r}, \ldots, v_{\omega}^{r}, r\right),
$$


see Figure 2(a). Observe that the line joining $v_{j}$ and $v_{j+1}^{f}$ either intersects the line segment $[f, r]$ or intersects some edge in $\lambda\left(v_{j}, r\right)$. If this line intersects $[f, r]$ then that contradicts the fact that $r$ is the closest point from $f$ in $[f, d]$ for which $\left|\lambda\left(u_{i}, r\right)\right|<\left|\lambda\left(u_{i}, f\right)\right|$. Hence, the line joining $v_{j}$ and $v_{j+1}^{f}$ intersects an edge of $\lambda\left(v_{j}, r\right)$ (see Figure 2(a)) at a point $\alpha$. From the convexity properties of geodesic paths and the triangle inequality, $\left|\lambda\left(v_{j}, v_{j+1}^{f}, \alpha\right)\right|<\left|\lambda\left(v_{j}, v_{j+1}^{r}, \alpha\right)\right|$. This contradicts the fact that the shortest path between $v_{j}$ and $r$ is via $v_{j+1}^{r}$. Hence the claim holds.

We continue with the proof of Lemma 1 . Let $j$ be the index such that $r$ is the intersection of $l_{i}$ and the line joining the two consecutive vertices $v_{j}$ and $v_{j+1}$ of $\lambda\left(u_{i}, f\right)$ (see Figure 2(b)). Denote the intersection between $l_{i}$ and the line joining $v_{j+1}$ and $v_{j+2}$ by $r^{*}$. Observe that $\left|\lambda\left(u_{i}, r^{*}\right)\right|<\left|\lambda\left(u_{i}, r\right)\right|$. This contradicts the fact that $r$ is the closest point from $f$ in $[f, d]$ for which $\left|\lambda\left(u_{i}, r\right)\right|<\left|\lambda\left(u_{i}, f\right)\right|$. Hence $r$ must be the intersection of $l_{i}$ and the line joining $a_{i}(f)$ and $b_{i}$, where $b_{i}$ is the vertex previous to $a_{i}(f)$ on the path $\lambda\left(u_{i}, f\right)$. But $\left|\lambda\left(a_{i}(f), f\right)\right|<\left|\lambda\left(a_{i}(f), r\right)\right|$. Therefore, $\left|\lambda\left(u_{i}, f\right)\right|<\left|\lambda\left(u_{i}, r\right)\right|$. Hence, we arrive at a contradiction.

For any anchor vertex $a_{i}(f)$, let $Z_{i}$ denote the set of points in $P$ which are at distance at most $\left|a_{i}(f) f\right|$ from $a_{i}(f)$.

Observation 2 For any two users $u_{i}$ and $u_{j}$, we have $\left(\Gamma_{i} \cap \Gamma_{j}\right) \backslash\{f\}=\emptyset$ if and only if $\left(Z_{i} \cap Z_{j}\right) \backslash\{f\}=\emptyset$.

Proof. Observe that $Z_{i} \subseteq \Gamma_{i}$ and $Z_{j} \subseteq \Gamma_{j}$. Therefore, if $\left(Z_{i} \cap Z_{j}\right) \backslash\{f\} \neq \emptyset$, then $\left(\Gamma_{i} \cap \Gamma_{j}\right) \backslash\{f\} \neq \emptyset$.

Assume that $\left(Z_{i} \cap Z_{j}\right) \backslash\{f\}=\emptyset$. Observe that both $Z_{i}$ and $Z_{j}$ contain the point $f$. Hence, both the circles $C_{i}$ and $C_{j}$ share the same tangent $l_{i j}$ passing through $f$. Now $l_{i j}$ divides $P$ into two disjoint subpolygons $P_{i}$ and $P_{j}$. Observe that $P_{i} \subset \Gamma_{i}$ and $P_{j} \subset \Gamma_{j}$. Therefore, $\left(\Gamma_{i} \cap \Gamma_{j}\right) \backslash\{f\}=\emptyset$.

Observation 3 For any placement of a facility $f$ by $\mathcal{P}_{1}$, an optimal placement of a facility by $\mathcal{P}_{2}$ is the point $\stackrel{s}{s} \neq f$ in $P$ that pierces the maximum number of regions among $\left\{Z_{1}, Z_{2}, \ldots Z_{n}\right\}$.

The arrangement of the regions $Z_{1}, Z_{2}, \ldots Z_{n}$ divides $P$ into cells. All points within the same cell pierce the same set of regions. Define the depth of a cell to be the number of regions pierced by any point in that cell. The cell with maximum depth contains $f$, because all regions $Z_{1}, Z_{2}, \ldots Z_{n}$ contain $f$.

Consider a circle $C_{\epsilon}$ with radius $\epsilon>0$ that is centered at $f$; this circle pierces all cells containing $f$ (see Figure $3(\mathrm{a})$ ). Observe that $C_{\epsilon} \cap P$ can be a set of disjoint subsets of $C_{\epsilon}$. If $f$ is in the interior of $P$, then we can choose $\epsilon$ such that $C_{\epsilon}$ is completely contained in the interior of $P$. If $f$ belongs to the boundary of $P$, then we can choose $\epsilon$ such that $C_{\epsilon} \cap P$ is a single connected subset of $C_{\epsilon}$ (see Figure 3(a) where $C_{\epsilon} \cap P$ consists of three disjoint sets $\mu_{1}, \mu_{2}$, and $\mu_{3}$ ).

Consider any optimal placement $s$ for $\mathcal{P}_{2}$. Let $\gamma$ be the cell that contains $s$. From the previous discussion, any point in $\gamma$ acts as an optimal placement for 


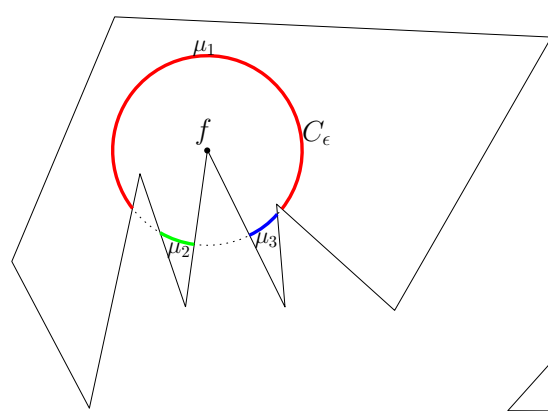

(a)

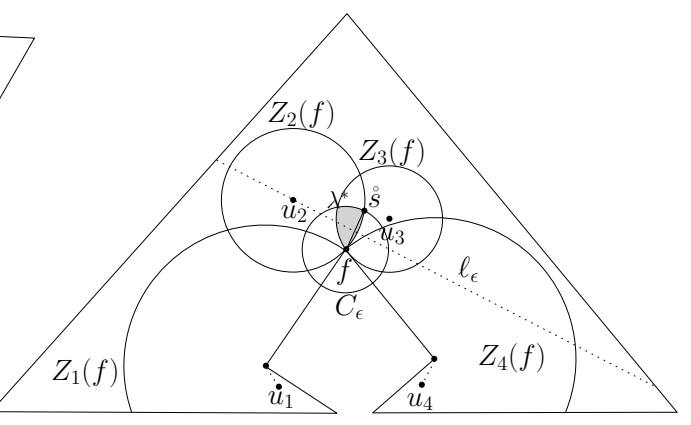

(b)

Fig. 3. Arrangement of the regions $\left\{Z_{1}, Z_{2}, \ldots Z_{n}\right\}$

$\mathcal{P}_{2}$. Hence, the intersection point between the boundary of $\gamma$ and $C_{\epsilon}$ is also an optimal placement. Thus, one of the optimal placements for $\mathcal{P}_{2}$ belongs to the set $\alpha_{\epsilon}=\left\{C_{i} \cap C_{\epsilon}: 1 \leq i \leq n\right\}$.

Consider any optimal placement of facility $\stackrel{s}{s} \alpha_{\epsilon}$ for $\mathcal{P}_{2}$. Let $\stackrel{s}{s}$ be the intersection point between $C_{i}$ and $C_{\epsilon}$. Consider the perpendicular bisector $\ell$ of $f$ and $\stackrel{s}{s}$. Let $\ell_{\epsilon} \subseteq \ell$ be the maximal line segment in $P$ that contains the midpoint of the line segment joining $f$ and $s$ (see Figure 3(b)).

Observation 4 The line segment $\ell_{\epsilon}$ passes through $a_{i}(f)$.

Proof. Observe that $[\stackrel{s}{s} f]$ is a chord of the circle $C_{i}$. The perpendicular bisector of any chord always passes through the center of the circle. Hence, the result holds.

Note that $\ell_{\epsilon}$ divides $P$ into two sub-polygons, one containing $f$ and the other containing $\stackrel{\circ}{s}$. If $\mathcal{P}_{2}$ places its facility at $\stackrel{\circ}{s}, \mathcal{P}_{2}$ will serve the set of users $u_{i}$ such that $a_{i}(f)$ belongs to the sub-polygon containing $\stackrel{\circ}{s}$. As $\epsilon$ tends to $0, \ell_{\epsilon}$ tends to the line joining $f$ and $a_{i}(f)$. Hence, for all $a_{i}(f)$, if we consider the chord passing through $f$ and $a_{i}(f)$, then we can find an optimal placement for $\mathcal{P}_{2}$. Note that all anchor vertices are visible from $f$. Thus, using an angular sorting, we can find an optimal placement for $\mathcal{P}_{2}$. We obtain the following result.

Theorem 1. Let $P$ be a polygon with $m$ vertices and let $\mathcal{U}$ be a set of $n$ pointusers in $P$. Given the placement of a point $f \in P$ by $\mathcal{P}_{1}$, a point $s \in P$ maximizing $\mathcal{P}_{2}$ 's payoff can be computed in $O(m+n(\log m+\log n))$ time.

Proof. Let $f$ be any placement of a facility by $\mathcal{P}_{1}$. Consider the visibility region $V_{f}$ of $f$ in $P$, i.e., the set of points which are visible from $f$. Observe that $P \backslash V_{f}$ consists of a set of possibly disjoint sub-polygons of $P$. For each such sub-polygon $P_{i}$, for all points $q \in P_{i}$, the anchor vertex on the path $\lambda(q, f)$ will be the same. Given $f$, we can construct a data structure in $O(m)$ time that can report the anchor vertex on the path $\lambda(q, f)$, for any query point $q \in P$, in $O(\log m)$ time [6]. Using this data structure, in $O(n \log m)$ time, we can find the 
set of all anchor vertices on the paths from users in $\mathcal{U}$ to $f$. Once we have the list of anchor vertices, using angular sorting, we can compute the half plane passing through $f$ which contains the maximum number of anchor vertices. Hence the result follows.

\section{Computing an Optimal Placement for $\mathcal{P}_{1}$}

As before, let $P$ be a simple polygon with $m$ vertices and let $\mathcal{U}=\left\{u_{1}, u_{2} \ldots u_{n}\right\}$ be a set of $n$ point-users in $P$. We will present an algorithm that computes an optimal placement of a facility for $\mathcal{P}_{1}$.

For any placement of $f$ by $\mathcal{P}_{1}$, let $\nu(f)=\max _{s}\left|\mathcal{U}_{f \cup s}(\{s\})\right|$, where the maximum is taken over all points $s$ in $P$ with $s \neq f$. Our objective is to find a point $\stackrel{f}{f} P$ which minimizes $\nu$; we call such a point an optimal placement for $\mathcal{P}_{1}$. Observe that there are two cases:

Case 1: $\stackrel{\circ}{f}$ belongs to the boundary of $P$.

Case 2: $f$ is in the interior of $P$.

In Section 3.1, we will give an algorithm that computes an optimal placement on the boundary of $P$ for $\mathcal{P}_{1}$. Formally, we will show how to compute a point $f_{b}$ on the boundary of $P$ such that $\nu\left(f_{b}\right)=\min _{f} \nu(f)$, where the minimum is taken over all points $f$ on the boundary of $P$. In Section 3.2, we will give an algorithm that computes an optimal placement in the interior of $P$ for $\mathcal{P}_{1}$.

\subsection{The boundary case}

Let us begin our discussion with the following two simple observations (see Figure 4).

Observation 5 For any placement $f$ by $\mathcal{P}_{1}$, where $f$ is on any convex vertex of $P$, there exists a placement $s$ for $\mathcal{P}_{2}$ such that $\nu(f)=n$.

Observation 6 Let $\left(v_{i}, v_{i+1}\right)$ be an edge of $P$ such that at least one of $v_{i}$ and $v_{i+1}$ is a convex vertex. For any placement $f$ by $\mathcal{P}_{1}$ on the edge $\left(v_{i}, v_{i+1}\right)$, there exists a placement $s$ for $\mathcal{P}_{2}$ such that $\nu(f)=n$.

Hence, an optimal placement for $\mathcal{P}_{1}$ must be either at a reflex vertex or on an edge $\left(v_{i}, v_{i+1}\right)$ for which both $v_{i}$ and $v_{i+1}$ are reflex vertices.

Observation 7 Let $\left(v_{i}, v_{i+1}\right)$ be an edge of $P$ such that both $v_{i}$ and $v_{i+1}$ are reflex vertices. For any placement $f$ by $\mathcal{P}_{1}$ on the edge $\left(v_{i}, v_{i+1}\right), \nu(f) \geq \nu\left(v_{i}\right)$ and $\nu(f) \geq \nu\left(v_{i+1}\right)$.

Proof. Let $p$ and $q$ be arbitrary placements by $\mathcal{P}_{1}$ and $\mathcal{P}_{2}$, respectively. The perpendicular bisector of $p$ and $q$ divides $P$ into two sub-polygons. Denote the sub-polygon that contains $p$ by $P^{+}(p, q)$, and the other sub-polygon by $P^{-}(p, q)$. 


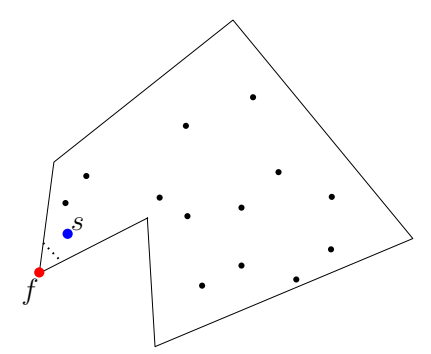

(a)

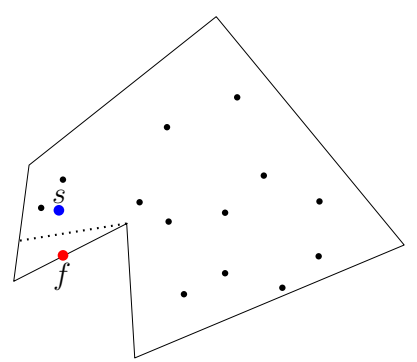

(b)

Fig. 4. (a) Illustration of Observation 5 (b) Illustration of Observation 6

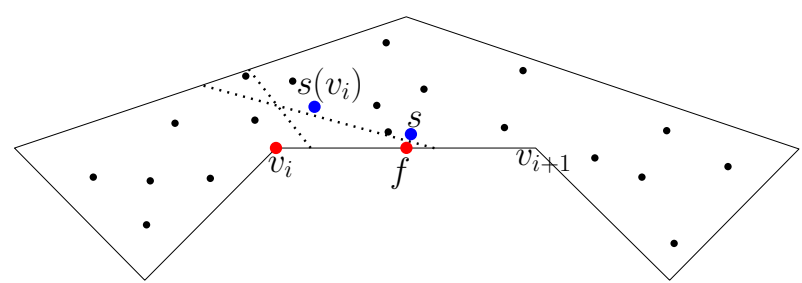

Fig. 5. Illustration of the proof of Observation 7

Let $f$ be any placement by $\mathcal{P}_{1}$ on the edge $\left(v_{i}, v_{i+1}\right)$ (see Figure 5 ). Let $s\left(v_{i}\right)$ be an optimal placement for $\mathcal{P}_{2}$, when $\mathcal{P}_{1}$ places its facility at $v_{i}$. Hence, $\nu\left(v_{i}\right)$ is the number of users in $P^{-}\left(v_{i}, s\left(v_{i}\right)\right)$. When $\mathcal{P}_{1}$ places its facility at $f$, there always exists a placement $s$ by $\mathcal{P}_{2}$, such that $s$ serves the set of users in $P^{-}\left(v_{i}, s\left(v_{i}\right)\right)$ (see Figure 5). Therefore, $\nu(f) \geq \nu\left(v_{i}\right)$ and the claim holds.

Thus, there is optimal placement, on the boundary of $P$, for $\mathcal{P}_{1}$ that is at a reflex vertex of $P$. By checking all reflex vertices, we can compute an optimal placement for $\mathcal{P}_{1}$ on the boundary of $P$ in $O\left(m^{2} \log m\right)$ time.

\subsection{The interior case}

In this section, we present an algorithm that computes an optimal placement for $\mathcal{P}_{1}$ in the interior of the polygon $P$. Let $R$ denote the set of reflex vertices of $P$. Consider the set $L$ of all maximal line segments which are fully contained in $P$ and contain at least two points from $R \cup \mathcal{U}$ (see Figure 6(a)). The set $L$ tessellates $P$ into a collection of cells. Denote the tessellation by $\Pi(P)$.

Recall the notion of an anchor vertex defined in Section 2.

Lemma 2. For any cell $C$ in $\Pi(P)$, for any two points $f_{1}$ and $f_{2}$ in $C$, and for any user $u_{i}$, we have $a_{i}\left(f_{1}\right)=a_{i}\left(f_{2}\right)$.

Proof. Assume there exists a user $u_{i}$ whose anchor vertex $a_{i}\left(f_{1}\right)$ on the geodesic path from $u_{i}$ to $f_{1}$ is different from the anchor vertex $a_{i}\left(f_{2}\right)$ on the geodesic path from $u_{i}$ to $f_{2}$. 


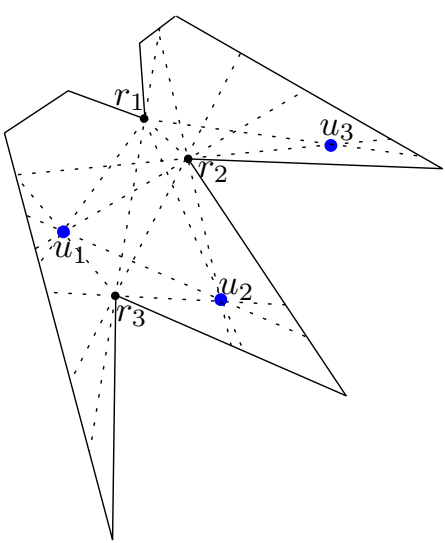

(a)

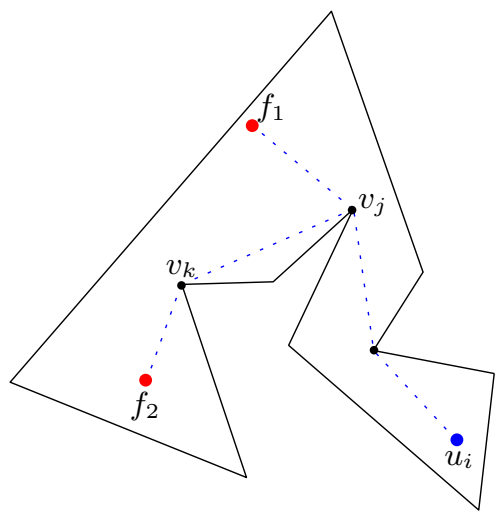

(b)

Fig. 6. (a) Tessellation of $P$ (b) Illustration of the proof of Lemma 2

Let $v_{j}$ be the last vertex that is common to the paths $\lambda\left(u_{i}, f_{1}\right)$ and $\lambda\left(u_{i}, f_{2}\right)$. Observe that one of $a_{i}\left(f_{1}\right)$ and $a_{i}\left(f_{2}\right)$ is not equal to $v_{j}$, because otherwise, we would have $a_{i}\left(f_{1}\right)=a_{i}\left(f_{2}\right)=v_{j}$. Assume, without loss of generality, that $a_{i}\left(f_{2}\right) \neq v_{j}$ (see Figure 6(b)).

Let $v_{k}$ be the vertex next to $v_{j}$ on the shortest path from $v_{j}$ to $f_{2}$. Observe that $f_{1}$ and $f_{2}$ are on different sides of the line joining $v_{j}$ and $v_{k}$. Hence, $f_{1}$ and $f_{2}$ belong to two different cells of the tessellation $\Pi(P)$.

Let $C$ be any cell in $\Pi(P)$. For any user $u_{i}$, all points $f$ in $C$ have the same anchor vertex; denote this anchor vertex by $a_{i}^{C}$. Assign a weight $w_{i}^{C}$ which is the number of shortest paths from any user $u_{j}$ to any point $f \in C$, in which $a_{i}^{C}$ is the anchor vertex.

Recall that a chord of $P$ is a closed line segment whose interior is contained in the interior of $P$ and whose endpoints are on the boundary of $P$. Let $f$ be any placement by $\mathcal{P}_{1}$. Any chord passing through $f$ divides $P$ into two sub-polygons, which we call half polygons with respect to $f$. From Section 2, we know that for any placement $f$ by $\mathcal{P}_{1}$, the maximum number of users that $\mathcal{P}_{2}$ can serve, by placing one facility, is equal to $\max _{P_{f}} \sum_{a_{i}^{C} \in P_{f}}\left|w_{i}^{C}\right|$, where $P_{f}$ is any half polygon with respect to $f$, i.e., the maximum number of anchor vertices in any half polygon with respect to $f$.

For any point $f$ in any cell $C$, we define the weighted half-space depth of $f$ to be $\max _{P_{f}} \sum w_{j}^{C}$ such that $a_{j} \in P_{f}$. Observe that an optimal placement for $\mathcal{P}_{1}$ in the cell $C$ corresponds to a point with minimum weighted half-space depth.

Lemma 3. One of the optimal placements for $\mathcal{P}_{1}$ belongs to the set of vertices of the tessellation $\Pi(P)$.

Proof. Assume that none of the optimal placements for $\mathcal{P}_{1}$ belongs to the set of vertices of $\Pi(P)$. Let $f$ be any optimal placement for $\mathcal{P}_{1}$. Suppose $f$ belongs to 


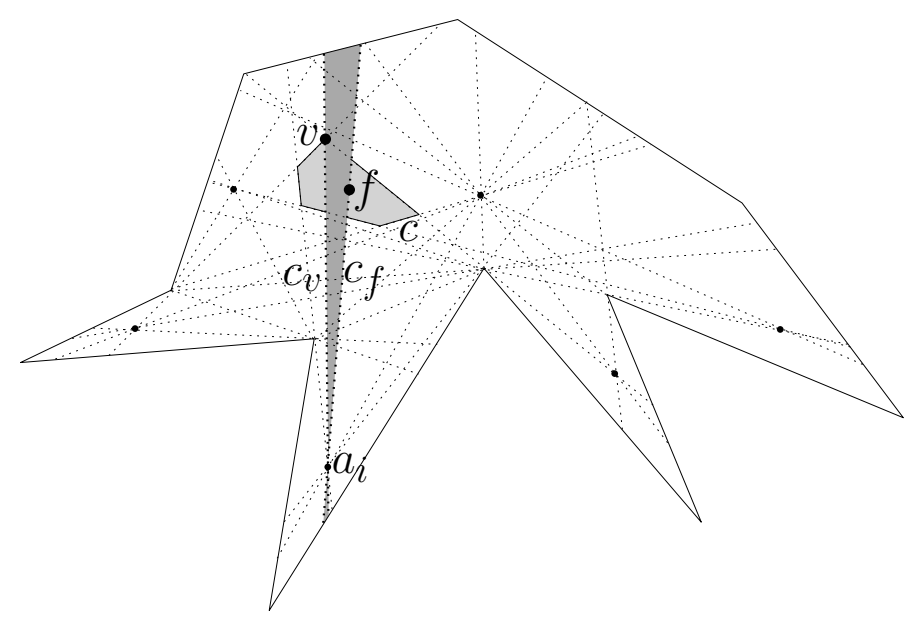

Fig. 7. Illustration of the proof of Lemma 3

the cell $C \in \Pi(P)$. Let $v$ any vertex of this cell. Let $\delta$ be the payoff of $\mathcal{P}_{1}$, when $\mathcal{P}_{1}$ places a facility at $v$, and assume that $\delta$ is less then the optimal payoff of $\mathcal{P}_{1}$. Then there exists a half-polygon $P_{v}$ bounded by a chord $c_{v}$, which contains $n-\delta$ users. With out loss of generality, we may assume that $c_{v}$ is passing through some anchor vertex $a_{j}$. Since $a_{j}$ is visible from $v, a_{j}$ is also visible from $f$. Consider the chord $c_{f}$ passing through $f$ and $a_{j}$ (see Figure 7 ). Consider the half polygon $P_{f}$ bounded by $c_{f}$. Observe that $P_{v} \backslash P_{f}=\emptyset$, because otherwise, $v$ and $f$ belong to different cells of $\Pi(P)$. It follows that the claim holds.

Since the cardinality of $R \cup \mathcal{U}$ is at most $n+m$, the number of cells and the number of vertices in the tessellation $\Pi(P)$ is $O\left((n+m)^{4}\right)$. For each vertex, we can check the optimal payoff of $\mathcal{P}_{1}$ in $O(m+n(\log n+\log m))$ time. Hence, we have proved the following result.

Theorem 2. Let $P$ be a polygon with $m$ vertices and let $\mathcal{U}$ be a set of $n$ pointusers in $P$. An optimal placement of a facility for $\mathcal{P}_{1}$ can be computed in polynomial time.

\section{Conclusion}

We have considered the Discrete Voronoi Game for a Simple Polygon $P$. The game consists of two players $\mathcal{P}_{1}$ and $\mathcal{P}_{2}$, and a finite set of users in a simple polygon $P$. Initially, $\mathcal{P}_{1}$ places one facility in $P$, after which $\mathcal{P}_{2}$ places another facility in $P$. Each user is then assigned to one of the facilities according to the nearest neighbor rule, where distances are measured using the geodesic distance in $P$. We have shown that an optimal strategy for $\mathcal{P}_{2}$, given any placement of $\mathcal{P}_{1}$, can be found in $O(m+n(\log m+\log n))$ time, and an optimal strategy for $\mathcal{P}_{1}$ can be found in polynomial time. 
There are many open problems in this area. Obtaining an algorithm to find an optimal placement for $\mathcal{P}_{1}$ and $\mathcal{P}_{2}$, where each of them places $k>1$ facilities is a problem that remains to be solved. Another variant of the game where the two players place $k>1$ facilities alternately is also an interesting problem to study.

\section{References}

1. Hee-Kap Ahn, Siu-Wing Cheng, Otfried Cheong, Mordecai J. Golin, and René van Oostrum. Competitive facility location: the Voronoi game. Theor. Comput. Sci., 310(1-3):457-467, 2004.

2. Sayan Bandyapadhyay, Aritra Banik, Sandip Das, and Hirak Sarkar. Voronoi game on graphs. In WALCOM, 2013, To appear.

3. Aritra Banik, Bhaswar B. Bhattacharya, and Sandip Das. Optimal strategies for the one-round discrete Voronoi game on a line. Journal of Combinatorial Optimization, pages $1-15,2012$.

4. Bhaswar B. Bhattacharya and Subhas C. Nandy. New variations of the maximum coverage facility location problem. European Journal of Operational Research, 224(3): $477-485,2013$.

5. Sergio Cabello, José Miguel Díaz-Báñez, Stefan Langerman, Carlos Seara, and Inmaculada Ventura. Facility location problems in the plane based on reverse nearest neighbor queries. European Journal of Operational Research, 202(1):99-106, 2010.

6. Leonidas J. Guibas and John Hershberger. Optimal shortest path queries in a simple polygon. J. Comput. Syst. Sci., 39(2):126-152, 1989.

7. S. L. Hakimi. On locating new facilities in a competitive environment. European Journal of Operational Research, 12 (1):29-35, 1983.

8. Sachio Teramoto, Erik D. Demaine, and Ryuhei Uehara. Voronoi game on graphs and its complexity. In Sushil J. Louis and Graham Kendall, editors, CIG, pages 265-271. IEEE, 2006. 